\title{
Avaliação hidráulica de um simulador de chuvas pendular ${ }^{1}$
}

\author{
Claudinei A. Montebeller ${ }^{2}$, Daniel F. de Carvalho ${ }^{3}$, Teodorico Alves Sobrinho ${ }^{4}$, Antonio C. da S. Nunes ${ }^{5} \&$ Emiliane Rubio $^{5}$ \\ 1 Trabalho financiado pelo CNPq \\ 2 UFRRJ, BR 475, Km 7, CEP 23851-970, Seropédica, RJ \\ ${ }^{3}$ DE/IT/UFRRJ. BR 465, km 7. Seropédica, RJ. CEP 23851-970. Fone: (21) 682-1865. E-mail:carvalho@ufrrj.br (Foto) \\ 4 DCA/UFMS - Campus de Dourados. E-mail: talves@ceud.ufms.br \\ 5 UFRRJ, BR 475, Km 7, CEP 23851-970, Seropédica, RJ
}

Protocolo $053-16 / 05 / 2000$

\begin{abstract}
Resumo: Com este trabalho, objetivou-se avaliar o diâmetro médio de gotas, a intensidade de precipitação e a uniformidade de distribuição de um simulador de chuvas, para diferentes combinações de diâmetro de bico e pressão de serviço. Após ter sido realizado o teste de diâmetro médio de gotas, foi utilizado um programa computacional, para se avaliar a relação entre a energia cinética da chuva simulada pelo equipamento e a energia da chuva natural; além disso, realizaram-se, também, testes de intensidade de precipitação e uniformidade de distribuição. Os resultados mostraram que a relação da energia cinética da chuva produzida pelo simulador e a energia cinética da chuva natural, apresentou valores acima de $75 \%$ quando foi utilizado o bico VeeJet 80.150 , para todas as pressões e intensidade de precipitação estudadas e que maiores coeficientes de uniformidade foram encontrados quando os bicos foram submetidos a maiores pressões de serviço.
\end{abstract}

Palavras-chave: infiltrômetro de aspersão, diâmetro de gota, intensidade de precipitação

\section{Hydraulic evaluation of a rainfall simulator}

\begin{abstract}
This research was developed with the objective of evaluating the average drop diameter, the rain intensity and the distribution uniformity, for different arrangements of nozzle diameters and service pressures of a suspended rainfall simulator. A computational program was utilized in order to evaluate the relationship between the rainfall kinetic energy simulated by a rainfall infiltrometer and natural rainfall kinetic energy. Beyond this, some tests of rainfall intensity and distribution uniformity were realized. The results showed the relation between the simulated rainfall kinetic energy and natural rainfall kinetic energy was higher than $75 \%$ when the VeeJet 80.150 nozzles were used, for all service pressures and rainfall intensities considered; and higher uniformity coefficient were obtained when the nozzles were submitted to higher service pressures.
\end{abstract}

Key words: sprinkler infiltrometer, drop diameter, rainfall intensity

\section{INTRODUÇÃO}

A irrigação por aspersão tem, como característica fundamental, a precipitação de água na forma de chuva artificial, por causa do fracionamento do jato d'água em gotas, o qual é conseguido pela passagem da água sob pressão em pequenos orifícios ou bocais. É um método muito empregado por adaptar-se a quase todos os tipos de cultura e topografia do terreno (Bernardo, 1995); entretanto, os aspersores empregados apresentam tamanho e alcance do jato variados, produzindo gotas de tamanho diferenciado que, conforme o diâmetro e velocidade, podem gerar a desagregação de partículas de solo, a qual constitui a primeira fase do processo erosivo; além disso, outras conseqüências não desejáveis podem ocorrer no processo de infiltração da água e no desenvolvimento das plantas (Matsura \& Santos, 1995; Pereira et al., 1996).
Pelo fato da infiltração constituir o processo pelo qual a água penetra no solo através de sua superfície, sua magnitude é severamente reduzida pela formação ou presença do selo superficial, fenômeno causado devido à pequena porosidade desta camada e à dominância de poros de pequeno diâmetro (Chaves et al., 1993). Segundo Reichert et al. (1992) a textura do solo afeta sensivelmente o salpico de partículas com o impacto das gotas de chuva, o que contribui para uma redução da porosidade da camada superficial do solo. Além do impacto da gota, Morin \& van Winkel (1996) citam a dispersão físico-química das argilas do solo como causas da formação do selamento superficial e, conseqüentemente, da redução da velocidade de infiltração.

Diversos equipamentos são utilizados na determinação da capacidade de infiltração de água no solo. Os infiltrômetros de aspersão ou simuladores de chuva, têm sido bastante utilizados 
pelos pesquisadores da área de engenharia de conservação de solo e água. Esses equipamentos aplicam água por aspersão, podendo ser controlados a intensidade de precipitação, o tamanho e a velocidade de impacto das gotas sobre a parcela do solo onde se deseja estudar as características de infiltração, escoamento superficial e produção de sedimentos. Bubenzer \& Meyer (1965) foram um dos pioneiros no desenvolvimento de simuladores de chuva para estudos de erosão em laboratório, e comentam que o uso desses equipamentos torna-se extremamente necessário na obtenção de dados de escoamento superficial e de erosão, em curtos períodos de tempo.

Apesar de, potencialmente, os simuladores de chuva serem considerados equipamentos importantes nesse estudo, eles devem, para isso, produzir gotas de diâmetro médio similar àquela da chuva natural, apresentar velocidade de impacto das gotas no solo o mais próximo possível da velocidade terminal das gotas de chuva, produzir precipitações com energia cinética próxima à da chuva natural e promover distribuição uniforme da precipitação sobre a parcela experimental em estudo (Meyer \& Harmon, 1979). Por isso, para que um simulador seja considerado satisfatório, Meyer \& McCune (1958) concluíram que o mesmo deve apresentar relação entre a energia cinética da precipitação simulada e aquela da chuva natural acima de $75 \%$. Além disso, diversos pesquisadores consideram que valores de CUC acima de $80 \%$ são aceitáveis para a uniformidade de distribuição em simuladores de chuva e infiltrômetros de aspersão.

Ciente da necessidade de se conhecer o funcionamento dos simuladores a fim de melhor simular a chuva natural, desenvolveu-se este trabalho com o objetivo de se avaliar o diâmetro médio de gotas, a intensidade de precipitação e a uniformidade de distribuição de um simulador de chuvas, para diferentes combinações de diâmetro de bico, pressão e regulagens da velocidade do ciclo do simulador.

\section{MATERIAL E MÉTODOS}

Este trabalho foi desenvolvido no Departamento de Engenharia da Universidade Federal Rural do Rio de Janeiro, localizada no município de Seropédica, RJ. Foi utilizado um simulador de chuvas portátil similar ao descrito por Meyer \& Harmon (1979) adquirido pelo Departamento, em um convênio internacional com a University of Maryland (USA). Realizados em laboratório, os testes permitiram avaliar-se o equipamento quanto ao diâmetro médio de gotas, a intensidade de precipitação e a uniformidade de distribuição do simulador equipado com bicos VeeJet 80.100 e 80.150 , produzidos pela Spraying Systems do Brasil, trabalhando sob diferentes condições de funcionamento. Na Figura 1 tem-se o desenho esquemático do equipamento utilizado neste trabalho, no qual se observa que o funcionamento do equipamento depende das regulagens do painel de controle que, por sua vez, apresenta um relógio de ciclo, responsável pelo controle da intensidade de precipitação.

Na determinação do diâmetro médio volumétrico de gotas foi utilizado o método da farinha, descrito por Oliveira (1991). Os testes foram realizados com os bicos posicionados a 2,87 m de altura e submetidos às pressões de 13,6, 20,4, 27,2 e 34,0 kPa, para regulagens do relógio de ciclo de $15,0 \mathrm{~s} \mathrm{ciclo}^{-1}$. A pressão de serviço (Ps) do bico foi medida a $0,05 \mathrm{~m}$ da saída do bocal, utilizando-se um manômetro antivibrante com saída angular, provido de mostrador de $108 \mathrm{~mm}$ e escala de 0 a $156 \mathrm{kPa}$. Para cada determinação foram utilizadas três bandejas com farinha de trigo, que foram passadas sob jato de gotas pulverizadas pelo bico, a 0,20 m da superfície do solo, para evitar respingos. As bandejas ficaram expostas à precipitação durante um período de 3 a 4 s aproximadamente. Após secados ao ar por um período de $24 \mathrm{~h}$, os grânulos foram separados por peneiramento, utilizando-se as peneiras $4 ; 2 ; 1 ; 0,5 ; 0,25$ e 0,105 mm e, depois, secos em estufa por $24 \mathrm{~h}\left(105-110^{\circ} \mathrm{C}\right)$ sendo, então, pesados em balança eletrônica, obtendo-se a massa média dos grânulos.

O diâmetro da gota, considerada esférica, foi determinado pela Eq. 1, abaixo:

$$
\mathrm{D}=\sqrt[3]{\frac{6 \mathrm{~m}}{\pi \rho}}
$$

em que:

$$
\begin{array}{ll}
\mathrm{D} & \text { - diâmetro da gota, } \mathrm{mm} \\
\mathrm{m} & \text { - massa média da gota, em } \mathrm{mg} \\
\rho & \text { - massa específica da água, } \mathrm{em} \mathrm{mg} \mathrm{mm}^{-3}
\end{array}
$$

O volume de água por peneira foi calculado multiplicando-se o número de gotas ou grânulos pelo volume da gota relativo a cada peneira. $\mathrm{O}$ volume de cada gota foi calculado pela Eq. 2:

$$
\mathrm{V}=\frac{\pi \mathrm{D}^{3}}{6}
$$

em que:

$$
\text { V - volume da gota d'água, } \mathrm{mm}^{3}
$$

Acumulando-se o volume de água por peneira em relação ao número médio de gotas retidas em cada peneira, foram confeccionados gráficos entre o volume de água acumulado e o diâmetro médio de gota retida em cada peneira, sendo possível determinar-se o D50, ou seja, o diâmetro médio apresentado por $50 \%$ das gotas.

De posse do D50 obtido para cada combinação de pressão de serviço e diâmetro do bico, foi possível avaliar-se a velocidade da gota e o seu efeito na energia cinética da chuva por meio de um programa computacional desenvolvido por Alves Sobrinho (1997) que permitiu selecionar a melhor combinação de pressão de serviço e velocidade do ciclo que produzisse um valor estimado da velocidade das gotas que mais se aproximasse da velocidade das gotas da chuva natural, conforme Laws \& Parsons (1943). Esses autores propuseram uma relação a ser utilizada para efeito de comparação da chuva natural com os dados de chuva simulada. Os valores de intensidade de precipitação em função do diâmetro volumétrico médio de gotas são apresentados na Tabela 1 .

A uniformidade de distribuição de água dos bicos foi calculada empregando-se o coeficiente de Christiansen (Eq. 3). Para obtenção do volume de água aplicado, utilizaram-se 25 copos coletores, distribuídos numa área de $1 \mathrm{~m}^{2}$. O tempo de aplicação de água foi de $3 \mathrm{~min}$, sendo que as pressões utilizadas foram as mesmas do teste da farinha e as regulagens do relógio de ciclo 


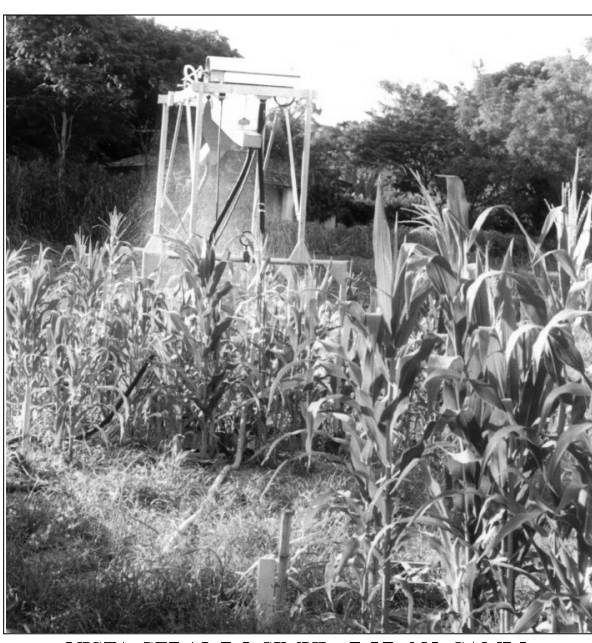

VISTA GERAL DO SIMULADOR NO CAMPO

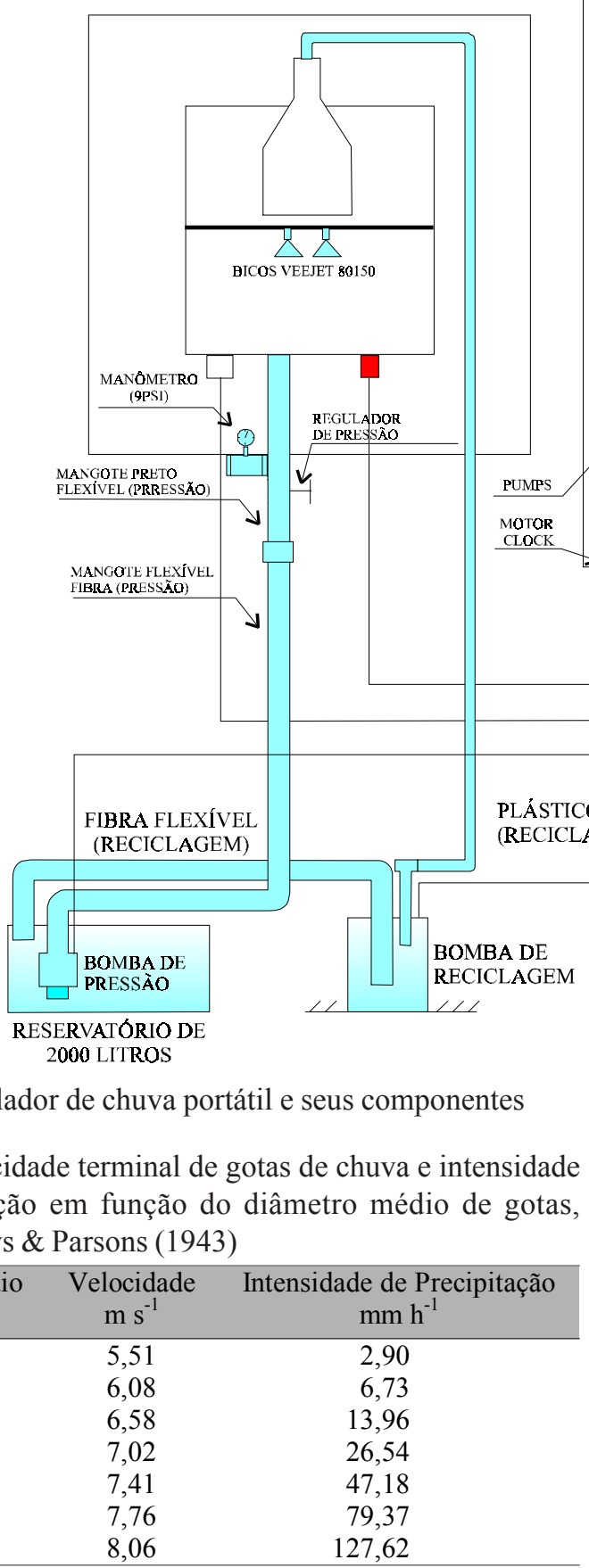

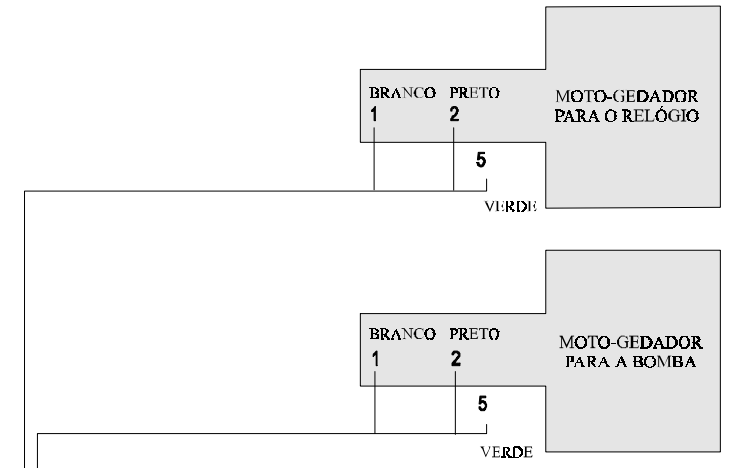

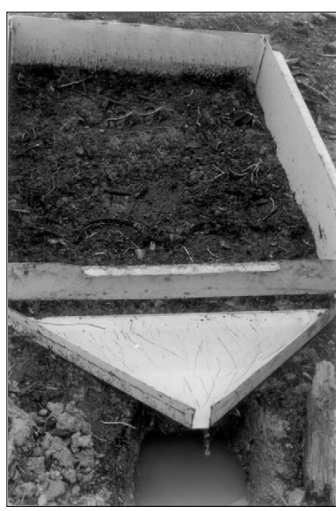

DETALHE DA CALHA

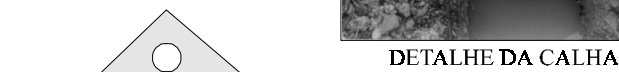

MOTOR $<$ MOZZLE

- ON $\square$ ON-OFF CLOCK

OFF ON-OFF MOTOR 7 ON-OFF DIGITAL

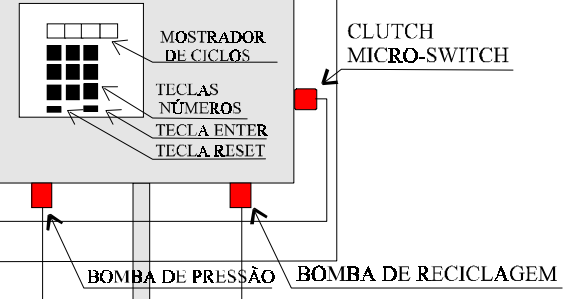

\section{VEL}

ÁSTICO FLEXTVEL
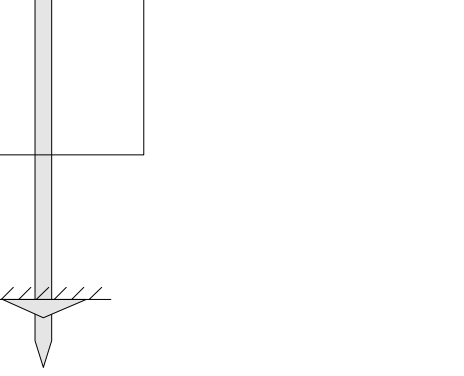

foram de 2 e $4 \mathrm{~s} \mathrm{ciclo}^{-1}$. Ao final de cada teste, os copos com os volumes de água coletados eram pesados em uma balança digital com precisão de 0,01 g. Em cada teste, a intensidade média de precipitação de água foi determinada pela relação entre a lâmina média de água obtida nos coletores e o tempo de aplicação.

$$
\mathrm{CUC}=100\left(1-\frac{\sum_{\mathrm{i}=1}^{\mathrm{n}}|\mathrm{Xi}-\overline{\mathrm{X}}|}{\mathrm{n} \overline{\mathrm{X}}}\right)
$$

em que:

CUC - coeficiente de uniformidade de Christiansen 
$\mathrm{X}_{\mathrm{i}}$ - lâmina precipitada em cada coletor

X - lâmina média precipitada

n - número de copo coletores

\section{RESULTADOS E DISCUSSÃO}

Na Figura 2 observam-se as curvas de volume acumulado versus diâmetro de gota, para os bicos VeeJet 80.100 e 80.150, à pressão de $13,6 \mathrm{kPa}$. Como o D50 se refere ao diâmetro da gota que corresponde a um volume acumulado de $50 \%$, percebe-se que o D50 foi menor quando se utilizou o bico 80.100 em relação ao bico 80.150 . Este comportamento era esperado, uma vez que, mantida a pressão, o bico com menor área de passagem irá promover maior pulverização do jato, proporcionando diâmetros de gotas menores.

(A)

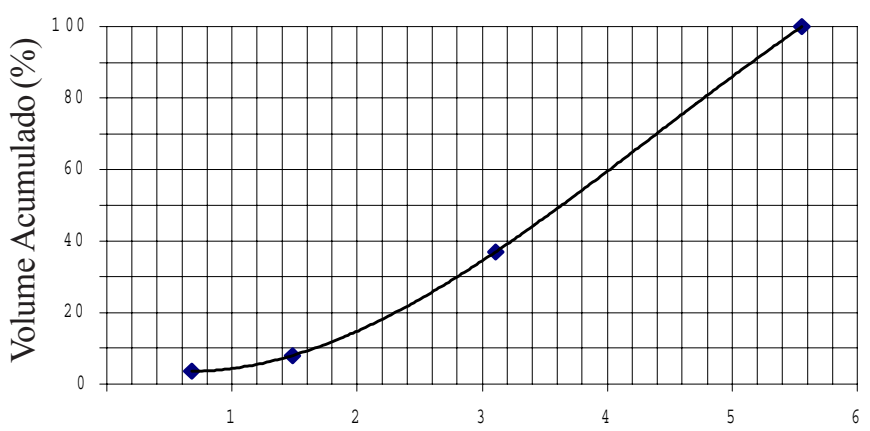

(B)

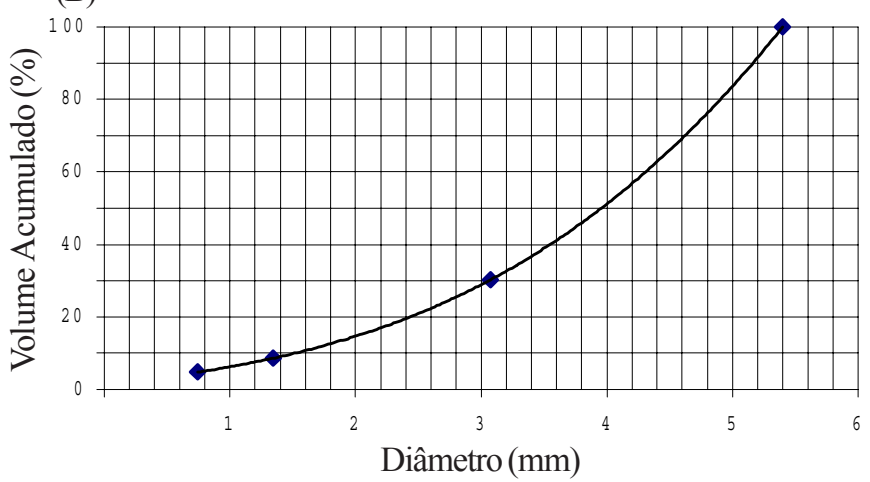

Figura 2. Volume acumulado versus diâmetro de gota paras os bicos VeeJet 80-100 (A) e VeeJet 80-150 (B), submetidos à pressão de $13,6 \mathrm{kPa}$

Os resultados finais do teste da farinha são apresentados na Tabela 2, para cada combinação de bocal e pressão utilizada; seguindo-se a mesma tendência para a mesma pressão de serviço, o D50 obtido para o bico 80.100 é inferior ao obtido para o bico 80.150 ; verifica-se, também, que a medida em que se aumenta a pressão, considerando-se o mesmo tamanho de bico, o D50 diminui em razão da maior pulverização do jato, fracionando-o em gotas de menor diâmetro. Este fracionamento é mais acentuado quando se utiliza o bico Veejet 80.100 , notadamente para as pressões de 20,4, 27,2 e 34,0 kPa, devido ao menor diâmetro do orifício de saída do jato.

Fazendo-se uso do programa computacional desenvolvido por Alves Sobrinho (1997) foi possível estimar-se a energia cinética da chuva produzida pelo simulador e compará-la
Tabela 2. Diâmetro médio de gotas (D50) obtidos com os bicos VeeJet 80.100 e 80.150 , para diferentes pressões de serviço

\begin{tabular}{ccc} 
Bico VeeJet & Pressão no Bico $(\mathrm{kPa})$ & D50 $(\mathrm{mm})$ \\
\multirow{3}{*}{80.100} & 13,6 & 3,6 \\
& 20,4 & 2,0 \\
& 27,2 & 1,7 \\
& 34,0 & 1,5 \\
80.150 & 13,6 & 3,9 \\
& 20,4 & 2,2 \\
& 27,2 & 2,0 \\
34,0 & 1,9
\end{tabular}

com a da chuva natural. A Figura 3 apresenta os resultados obtidos para os bicos VeeJet 80.100 e 80.150 . A relação entre a energia cinética da chuva produzida pelo simulador e a energia cinética da chuva natural, correspondeu a valores acima de $75 \%$ apenas para intensidade de precipitação de até $30 \mathrm{~mm} \mathrm{~h}^{-1}$, para as pressões estudadas (Figura 3A); entretanto, para a pressão de $13,6 \mathrm{kPa}$, esta relação ficou acima de $85 \%$ em toda a faixa de intensidade de precipitação estudada.

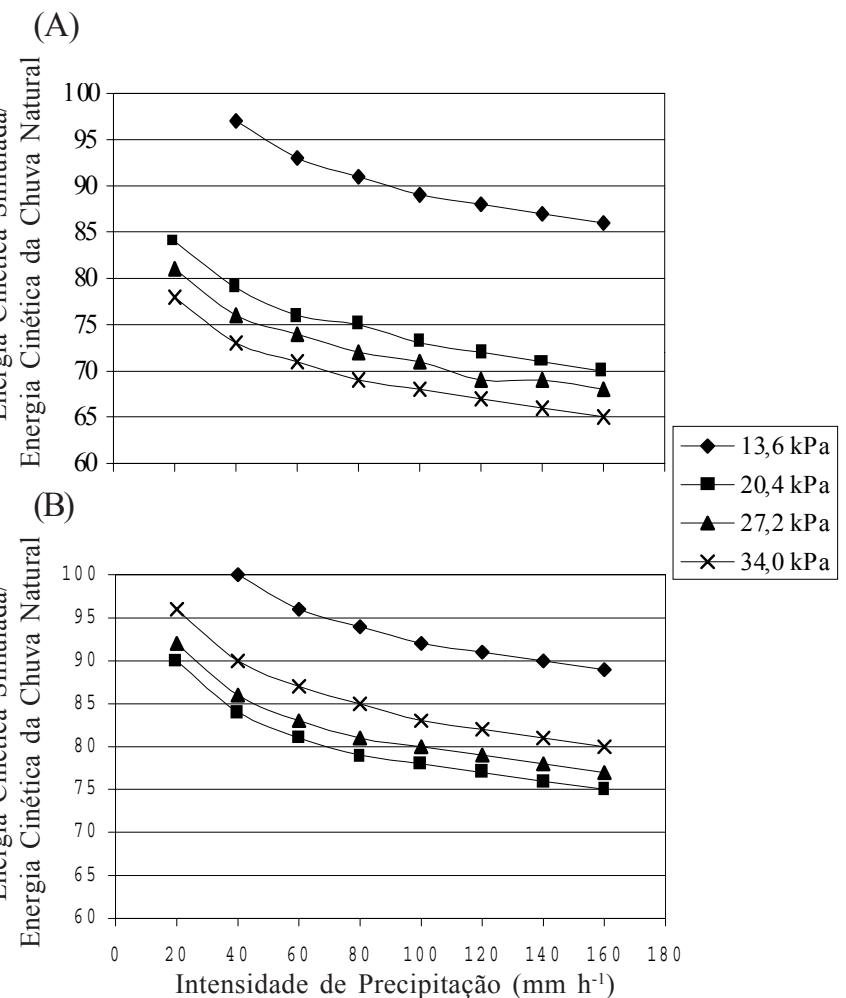

Figura 3. Relação entre a energia cinética da chuva produzida pelo simulador, utilizando-se os bicos VeeJet 80-100 (A) e VeeJet 80-150 (B), e energia cinética da chuva natural, em função da intensidade de precipitação

Analisando-se a Figura 3B, verifica-se que, através do bico VeeJet 80.150 , a relação entre a energia cinética da chuva produzida pelo simulador e a energia cinética da chuva natural, correspondeu a valores acima de $75 \%$ para todas as pressões e intensidades de precipitação estudadas. Percebe-se, com isto que, utilizando-se o maior bico, pode-se trabalhar numa faixa maior de valores para pressão, a fỉm de se obter relações de energia cinética superiores a 75\%. Alves Sobrinho (1997) ao desenvolver um simulador de chuvas com dispositivo rotativo 
para controle da intensidade de precipitação e trabalhando com pressão de $35,6 \mathrm{kPa}$, encontrou relação acima de $90 \%$, para precipitação de até $100 \mathrm{~mm} \mathrm{~h}^{-1}$.

Os valores da relação entre as energias cinéticas, utilizando-se o bico Veejet 80.100 a pressão de $34,0 \mathrm{kPa}$, foram inferiores aos valores obtidos para pressão de serviço de 13,6, 20,4 e 27,2 kPa (Figura 3A); no entanto, o mesmo não ocorreu quando se observaram as curvas apresentadas na Figura $3 \mathrm{~B}$, relativas ao bico Veejet 80.150 , o que pode ser explicado devido ao maior fracionamento das gotas, promovido pelo bico 80.100 , e à menor variação do diâmetro das gotas obtidas com o bico 80.150, operando com pressão acima de $20 \mathrm{kPa}$; este comportamento evidencia a importância da pressão de serviço na velocidade de impacto das gotas, associada ao diâmetro das mesmas.

Os resultados dos testes de intensidade de precipitação e uniformidade de distribuição, para o simulador equipado com os bicos 80.100 e 80.150, quando submetidos às pressões de $13,6,20,4,27,2$ e $34,0 \mathrm{kPa}$, encontram-se na Tabela 3.

Tabela 3. Valores de intensidade de precipitação e coeficiente de uniformidade de Christiansen (CUC) obtidos com os bicos 80.100 e 80.150 , para diferentes pressões de serviço

\begin{tabular}{cccc}
\hline Bico VeeJet & $\begin{array}{c}\text { Pressão } \\
(\mathrm{kPa})\end{array}$ & $\begin{array}{c}\mathrm{Ip}^{*} \\
\left(\mathrm{~mm} \mathrm{~h}^{-1}\right)\end{array}$ & $\begin{array}{c}\text { CUC } \\
(\%)\end{array}$ \\
\hline \multirow{3}{*}{80.100} & 13,6 & 34,2 & 29 \\
& 20,4 & 40,9 & 69 \\
& 27,2 & 40,5 & 81 \\
\multirow{3}{*}{80.150} & 34,0 & 42,5 & 88 \\
& 13,6 & 52,1 & 31 \\
& 20,4 & 49,3 & 66 \\
& 27,2 & 52,2 & 75 \\
& 34,0 & 53,6 & 84 \\
\hline
\end{tabular}

Valores referentes à regulagem do relógio de ciclos, para $2 \mathrm{~s}$ por ciclo

Os testes de intensidade de precipitação (Ip) apresentados, referem-se à regulagem do relógio de ciclos de $2 \mathrm{~s} \mathrm{ciclo}^{-1}$. Para esta regulagem, os valores de Ip variaram de 34,2 a $42,5 \mathrm{~mm} \mathrm{~h}^{-1}$ para as pressões variando de 13,6 a $34,0 \mathrm{kPa}$, utilizando-se o bico 80.100; entretanto, ao se utilizar o bico 80.150 , praticamente não foram encontradas diferenças na Ip para os valores de pressão adotados. Com relação à uniformidade, verifica-se que, à medida que se aumenta a pressão de serviço do bico, o coeficiente de uniformidade de Christiansen (CUC) também aumenta sensivelmente, atingindo valores superiores a $80 \%$ para o bico 80.100 , nas pressões de 27,2 e $34,0 \mathrm{kPa}$ e para o bico 80.150 , na pressão de $34,0 \mathrm{kPa}$. Este aumento dos valores do CUC está associado, de certa maneira, ao maior fracionamento das gotas com o aumento da pressão de serviço.

Os resultados apresentados mostram que a utilização do Bico 80.100 é bastante limitada, pois uma ampla faixa de Ip é obtida apenas para D50 de 3,6 $\mathrm{mm}(\mathrm{Ps}=13,6 \mathrm{kPa})$ mas apresenta CUC de $29 \%$. Para este bico, CUC acima de $80 \%$ só foi obtido para D50 menor que $1,7 \mathrm{~mm}$, correspondendo à Ps maior que $27,2 \mathrm{kPa}$.

Para o bico 80.150, nota-se que a sua utilização não é limitada pelo D50, uma vez que a relação entre a energia cinética da chuva produzida pelo simulador e a energia cinética da chuva natural se manteve acima de $75 \%$ para intensidades de até $160 \mathrm{~mm} \mathrm{~h}^{-1}$; entretanto, analisando-se o coeficiente de uniformidade, percebe-se que valores dessa variável acima de
$80 \%$ são obtidos apenas para Ps maiores que 27,2 kPa, proporcionando relação entre a energia cinética da chuva produzida pelo simulador e a energia cinética da chuva natural acima de $80 \%$ para Ip de até 160 mm.h. ${ }^{-1}$.

\section{CONCLUSÕES}

Com base nos resultados obtidos, conclui-se que:

1. O simulador de chuvas avaliado apresentou desempenho satisfatório com relação às variáveis estudadas.

2. Maiores coeficientes de uniformidade foram encontrados quando os bicos foram submetidos a maiores pressões de serviço.

3. Comparando-se os resultados obtidos com os valores recomendados para as variáveis estudadas, conclui-se que o simulador avaliado deve trabalhar com bico 80.150 , a pressões de serviço superiores a $27,2 \mathrm{kPa}$ e intensidade de precipitação inferior a $160 \mathrm{~mm} \mathrm{~h}^{-1}$.

\section{LITERATURA CITADA}

Alves Sobrinho, T. Desenvolvimento de um infiltrômetro de aspersão portátil. Viçosa, MG: UFV, 1997, 85 p. Tese Doutorado

Bernardo, S. Manual de irrigação. 6. ed. Viçosa, MG: UFV, 1995. $657 \mathrm{p}$.

Bubenzer, G.D.; Meyer, L.D. Simulation of rainfall and soils for laboratory research. Transaction of the ASAE, St. Joseph, v.8, n.1, p.73-75, 1965.

Chaves, H.M L; Orlowski, E.; Roloff, G. Previsão da infiltração sob condições dinâmicas de selamento superficial. Revista Brasileira de Ciência do Solo, Campinas, v.17, n.2, p.141-147, 1993.

Laws, J.O.; Parsons, D.A. The relation of rain drop size to intensity. Transaction, American Geophysical Union, Washington, v.24, p.452-459, 1943.

Matsura, E.E.; Santos, A.H.P. Efeito do impacto de gotas de um aspersor no selamento superficial. In: Congresso Brasileiro de Engenharia Agrícola, 24, Viçosa, 1995. Anais... Viçosa: SBEA, 1995, p.224.

Meyer, L.D; Harmon, W.C. Multiple intensity rainfall simulator for erosion research on row sideslopes. Transactions of the ASAE, St. Joseph, v.22, n.1, p.100-103, 1979.

Meyer, L.D; McCune, D.L. Rainfall simulator for runoff plots. Agricultural Engineering, St. Joseph, v.39, n.1, p.644-648, 1958.

Morin, J; van Winkel, J. The effect of raindrop impact and sheet erosion on infiltration rate and crust formation. Soil Science Society of America Journal, v.60, p.1223-1227, 1996.

Oliveira, R.A. Distribuição de gotas por tamanho e perfil de precipitação de um aspersor fixo. Viçosa, MG: UFV, 1991, 103p. Dissertação Mestrado

Pereira, G.M; Lima, L.A.; Muniz, J. A. Impacto da irrigação por aspersão sobre a queda de flores do feijoeiro (Phaseolus vulgaris L.) e sobre o salpico do solo. In: Congreso Latinoamericano de Ingeníeria Agrícola, 2, Bauru, 1996. Anais... Bauru: SBEA, 1996, p.161.

Reichert, J.M; Veiga, M; Cabeda, M.S.V. Selamento superficial e infiltração de água em solos do Rio Grande do Sul. Revista Brasileira de Ciência do Solo, Campinas, v.16, n.3, p.289-298, 1992. 